\title{
Event-Detection Algorithms for Low Sampling Non-Intrusive Load Monitoring Systems based on Low Complexity Statistical Features
}

\author{
Attique Ur Rehman, IEEE Graduate Student Member, Tek Tjing Lie, Senior IEEE Member, Brice \\ Vallès, and Shafiqur Rahman Tito, IEEE Member
}

\begin{abstract}
One of the key techniques towards energy efficiency and conservation is Non-Intrusive Load Monitoring (NILM) which lies in the domain of energy monitoring. Event detection is a core component of event-based NILM systems. This paper proposes two new low-complexity and computationally fast algorithms that detect the variations of load data and return the time occurrences of the corresponding events. The proposed algorithms are based on the phenomenon of a sliding window that tracks the statistical features of the acquired aggregated load data. The performance of the proposed algorithms is evaluated using real-world data and a comparative analysis has been carried out with one of the recently proposed event detection algorithms. Based on the simulations and sensitivity analysis it is shown that the proposed algorithm can provide the results of up to $93 \%$ and $88 \%$ in terms of recall and precision respectively.
\end{abstract}

Index Terms-Energy Monitoring, Event Detection, Non-Intrusive Load Monitoring, Smart Grids.

\section{INTRODUCTION}

$\mathrm{E}$ NERGY efficiency and conservation are the key drivers towards the concept of the future smart grid (SG). The consumers who are expected to play a key role in this regard [1] not only can effectively participate towards the sustainable SG system but also can have direct feedback of meaningful real-time appliance level consumption information $[2,3]$. Due to the worldwide deployment of smart meters, energy monitoring becomes more viable today. Numerous applications based on smart meter data have already been reviewed [4] for the application of SG in achieving load diversity and efficiency [5].

Energy disaggregation, also known as load or power disaggregation [6], contributes to the development of effective energy monitoring systems. This refers to a method aiming at an estimation of individual appliances' power consumption from the aggregated household electricity consumption. Numerous methods are available to perform load disaggregation. They can be broadly distinguished as hardware and software-based methods [3, 7] as shown in Fig. 1.

Intrusive Load Monitoring (ILM) and smart appliances are the techniques that lie within the domain of hardware methods whilst Non-Intrusive Load Monitoring (NILM) lies in the domain of software methods. ILM refers to a technique in which appliance level power consumption profiles are obtained using sub-metering sensors that are attached to individual appliances [8]. This technique is relatively simple but factors like multiple numbers of sensors, reliability, and cost can be some of the main deterrent concerns [7, 9]. Smart appliances are the appliances that have integrated capabilities to monitor and report their power consumption [10] but these appliances are not widely in use due to their high market prices and interoperability issues [11]. Alternatively, software methods provide attractive solutions to load disaggregation. A widely used technique is commonly referred as NILM or Non-intrusive Appliance Load Monitoring (NALM) or Non-Intrusive Appliance Load Monitoring (NIALM) [12-14]. The concept was first introduced by Hart [15] in 1984. Later, numerous techniques have been proposed that improve the early concept of NILM. A comprehensive review and outlook of the proposed NILM algorithms are presented in [3, 16-18].

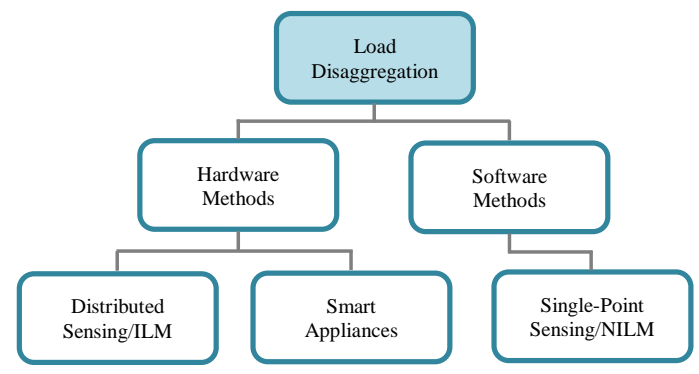

Fig. 1. Load Disaggregation Hierarchy

\section{A. Non-Intrusive Load Monitoring}

NILM is a widely used technique that disaggregates the load data acquired from a single entry point and identifies power consumption profiles of the individual appliances. Let consider a time-series power load curve monitored at a metering point. It can be represented as an algebraic sum of $n$ number of appliances' load, as shown in Eq. (1).

$$
P_{a g g}(t)=\sum_{i=1}^{n} P_{i}(t)
$$

The task of NILM is to identify the state of individual appliance loads $P_{l}(t), P_{2}(t), \ldots$ from the given information of aggregated load $P_{\text {agg }}(t)$. A traditional NILM system consists of three main components, namely: data acquisition, feature extraction, and classification as shown in Fig. 2. Additional

Attique Ur Rehman and Tek Tjing Lie are with Auckland University of Technology, New Zealand (Email: attique.rehman@aut.ac.nz| tek.lie@aut.ac.nz)

Brice Vallès is with Genesis Energy Ltd. New Zealand (E-mail: brice.valles@ genesisenergy.co.nz)

Shafiqur Rahman Tito is with Manukau Institute of Technology, New Zealand (Email: shafiqur.tito@manukau.ac.nz) 
details can be found in [3].

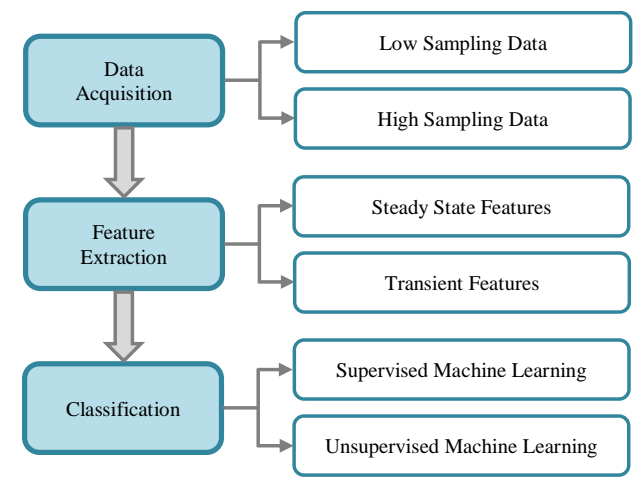

Fig. 2. Traditional NILM System Framework

Acquisition of the aggregated load data is a pre-requisite for any of the NILM systems. The aggregated load data can be composed of different variables (power, current, voltage, power factor etc.), and sampling rates. Feature extraction is a process to extract unique features (also referred to as signatures) from the acquired data. It can be broadly divided into two classes namely steady state and transient features. Both steady state and transient features are intended to identify the state changes in the operation of an appliance but differ in what data they are focusing on. The last component of the NILM system is classification and refers to the process of analyzing the features extracted from the acquired aggregated load data to identify specific appliances.

Early NILM research was focused on the disaggregation of high consumption loads [19-21]. Recently many of the algorithms are mainly focusing on high sampling rates [22] giving the opportunity to incorporate more appliance's electrical features [23]. This leads to accurately classify a greater number of appliances [2]. High and low sampling rates lead to the extraction of transient and steady-state features respectively. Armel et al. [2] presented a comprehensive analysis of the sampling rate (at which the aggregate data are acquired) and the corresponding number of appliances to be classified.

The working principle of the available NILM systems can be classified to be either event-based or non-event based [24] working principle of the method. The event-based NILM relies on event detection by using different edge detection algorithms on the acquired aggregated load data. Later-on features are captured from the extracted events and classified by a different set of rules using machine-learning algorithms. Figueiredo et al. [25] successfully detected steady state step changes, and features were classified by means of machine learning algorithms. On the other hand, the non-event based NILM does not rely on edge detection algorithms before the classification stage. Rather, all the samples of the acquired aggregated load data are considered for inference using statistical models such as Hidden Markov Model (HMM) [26-28]. A comparison between event-based and non-event based NILM is presented in Table I [7].

\section{B. Event-Based NILM}

An event is a fragment of a signal that deviates from the previous steady state and lasts until the next steady state has
TABLE I. COMPARISON OF EVENT-BASED AND NON-EVENT BASED NILM

\begin{tabular}{|l|l|}
\hline Event-Based & Non-Event Based \\
\hline $\begin{array}{l}\text { It is computationally efficient } \\
\text { due to event detection inference } \\
\text { carried out only on the detected } \\
\text { events. }\end{array}$ & $\begin{array}{l}\text { Non-event based inference is } \\
\text { carried out on all samples of the } \\
\text { data. Hence, computational cost } \\
\text { increases [29-31]. }\end{array}$ \\
\hline $\begin{array}{l}\text { Events' false detection or } \\
\text { misdetection may lead to errors. }\end{array}$ & $\begin{array}{l}\text { Wrong estimation errors for a } \\
\text { given sample can be corrected. }\end{array}$ \\
\hline
\end{tabular}

been reached [32]. Here it is worth mentioning that the turning $\mathrm{ON}$ and turning OFF of an appliance are considered as distinct events having their own starting and ending time respectively, as shown in Fig. 3.

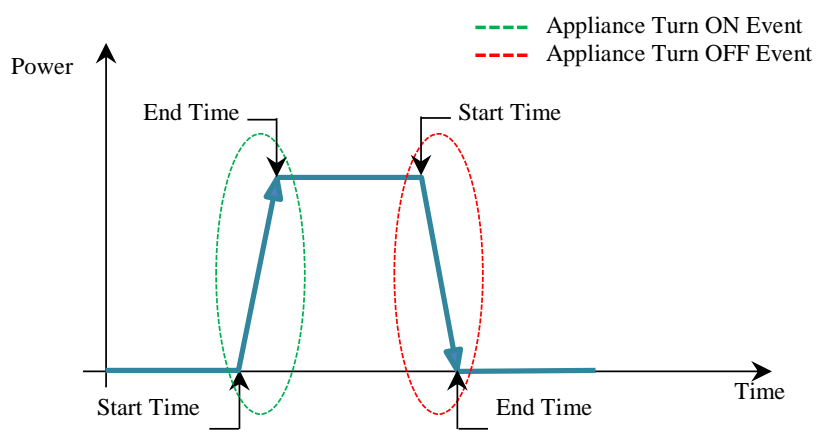

Fig. 3. Graphical Representation of an Event

The purpose of the event detection algorithm is to identify all turning $\mathrm{ON}$ and $\mathrm{OFF}$ edges of appliances within the acquired aggregated load data. A traditional event-based NILM system consists of data acquisition, data pre-processing, edge detection, feature extraction, and appliance classification [14]. After the aggregated load data are acquired, data pre-processing can be carried out to tackle power (load) measurement uncertainties. Data pre-processing can be performed either in the form of power normalization, thresholding, or filtering. The former aims at preventing uncertainties arising from data fluctuation that could cause misleading appliances' events. Thresholding aims at eliminating small power loads and the base-load from appliances that are running permanently and would both appear as noise. Lastly filtering is used for data smoothing and eliminating sudden peaks [13]. Later, edge detection is carried out to identify the events of appliances' turning ON and OFF. This is followed by the features extraction stage and finally, classification is done based on the extracted features.

To date, numerous event detection algorithms have been proposed and developed with diversity in terms of variables, data granularity, and techniques. Most of the existing work is based on appliance consumed power as an input variable with some exceptions, e.g., current harmonics used as an input feature to detect the events [32, 33]. De Baets et al. [29] performed event detection in the frequency domain by taking active power as input feature at a sampling rate of $60 \mathrm{~Hz}$. Girmay et al. [34] proposed a time-frequency based event detection using a goodness-of-fit Chi-squared test.

This paper proposes two low complexity and computationally fast event detection algorithms based on two 
different statistical features, namely variance and mean absolute deviation. The remaining of this paper is structured as follows: Section II presents the detailed phenomenon and working principle of the proposed algorithms; Section III presents the simulation studies and the corresponding results along with the performance evaluation. Sections IV and V present sensitivity and comparative analysis of the proposed algorithms respectively. Conclusions are drawn in Section VI.

\section{PRoposed AlgorithMS}

This section presents the proposed algorithms with details of their basic working principles and implementation. The basic principle of both algorithms relies on a sliding window that runs over the acquired aggregated load data to extract distinct events. This is graphically presented in Fig. 4.

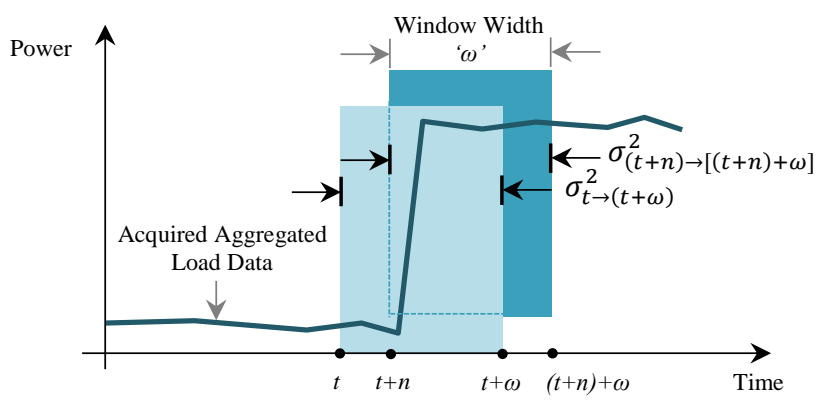

Fig. 4. Working Principle of the Proposed Algorithms

The sliding window of each algorithm tracks different statistical measures namely variance ' $\sigma^{2}$ ' and mean absolute deviation ' $M A D$ '. These measures are described in (2) and (3) respectively,

$\sigma^{2}=\frac{1}{n} \sum_{i=1}^{n}\left|x_{i}-\mu_{x}\right|^{2}$

$M A D=\frac{1}{n} \sum_{i=1}^{n}\left|\boldsymbol{x}_{i}-\mu_{x}\right|$

where $\mu_{x}$ is the mean of $\boldsymbol{x}$ as shown below in (4)

$\mu=\frac{1}{n} \sum_{i=1}^{n} x_{i}$

The proposed algorithms are based on variance and mean absolute deviation and are then called hereafter Variance Sliding Window (VSW) and Mean Absolute Deviation Sliding Window (MAD-SW) algorithms respectively. The output of both proposed algorithms is in the form of starting and ending time indices of the detected events from the acquired aggregated load data. The descriptions of the proposed algorithms are as follows:

\section{Algorithm}

\section{Input: Aggregated Load Data \\ Output: Start and End Time Indices of Detected Events}

1. Acquire aggregated load data and process the data using median filtering technique

2. Select the sliding window width ' $\omega$ '
3. Initialize the filter with the corresponding statistical feature, i.e., $\boldsymbol{\sigma}^{2}$ or $M A D$ (depending on the applied algorithm)

4. Using sliding window technique, compute iteratively the corresponding statistical features, i.e., $\boldsymbol{\sigma}^{2}$ or $M A D$ (depending on the applied algorithm)

5. Select a threshold value ' $\delta$ ' and compute the threshold signal representing the steady states and transient states

6. Use the derivation function to compute the corresponding edges and extract the starting and ending time instances of the detected events

7. Post-processing, i.e., event approval and delay correction due to window width

Detailed steps of the proposed algorithms are represented graphically in form of a flowchart as shown in Fig. 5.

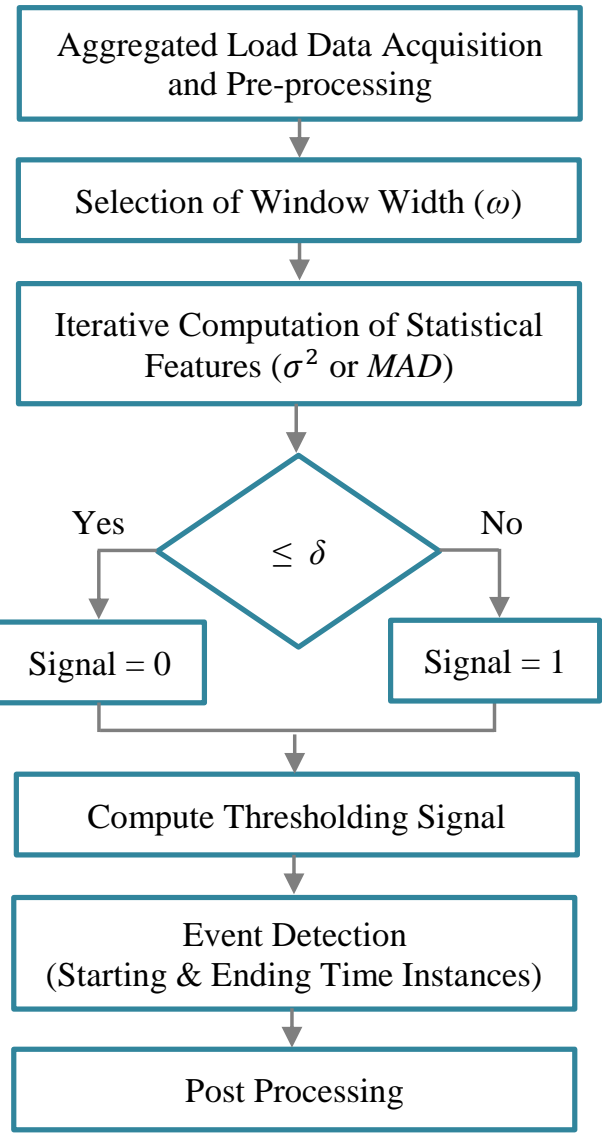

Fig. 5. Flow Chart of the Proposed Algorithms

\section{Simulation AND RESUlts}

For a realistic testing of the proposed algorithms, simulation studies have been carried out based on a comprehensive realworld dataset: Dataport [35]. Dataport is the largest load disaggregation data source, operated and owned by Pecan Street Inc., a non-profit research institute founded in 2009. Dataport is comprised of electricity consumption profiles for 722 houses [36] in the United States of America at a low sampling rate of 1 minute. Every house consists of aggregated average power consumption data as well as power consumption profiles of more than ten different appliances including but not limited to, 
air condition, microwave, dishwasher, oven, furnace, refrigerator etc. Furthermore, the ground-truth ${ }^{1}$ power signals of the said appliances are also available in Dataport.

\section{A. Simulation Parameters}

Simulations have been carried out on a total of 360 hours (15 days) of aggregated load data acquired from Dataport. MATLAB has been used as the primary computational tool. As the simulations are run on real-world data, (aggregated) power measurement uncertainties need to be taken care of before applying the event detection algorithm. In this paper, power measurement uncertainties in form of noise/data spikes are considered. In order to remove these spikes, for avoiding any interference with event detection, median filtering is used as a data pre-processing technique [37]. The threshold value ' $\delta$ ' has been selected to $250 \mathrm{~W}$. This value leads to the reduction of errors due to minor fluctuations in the acquired aggregated load data. It is also more viable for the events detection of high consumption appliances such as Electric Vehicle (EV) charging and Air Conditioning (AC).

To define the needed accuracy of the proposed algorithm to perform well, a parameter named as a delay tolerance ' $\Delta t$ ' is introduced. A detected event will be considered as a true positive if and only if $\left|t_{g}-t_{d}\right| \leq \Delta t$, where $t_{g}$ is the starting time of ground-truth event and $t_{d}$ is the starting time of the detected event by the proposed algorithm. Sensitivity analysis on delay tolerance has not been carried out in the present simulations and $\Delta t$ has been set equal to zero. Similarly, for the presented simulations and corresponding results, the window width has been kept constant to 5 samples. A comprehensive sensitivity analysis in terms of window width will be discussed in Section IV. Table II presents various parameters regarding Dataport and the proposed algorithms used for the simulation studies and results.

TABLE II. SIMULATION PARAMETERS REGARDING DATAPORT AND ALGORITHMS

\begin{tabular}{|l|l|}
\hline Dataport Data ID & 26 \\
\hline Data Timeframe & June 18 - July 2, 2014 \\
\hline Sampling Rate & $1 / 60 \mathrm{~Hz}$ (1 minute) \\
\hline No. of Data Samples & 21600 \\
\hline Window Width ' $\boldsymbol{\omega}$ ' & 5 samples \\
\hline Pre-processing Technique & Filtering (Median Filter) \\
\hline Threshold Value ' $\boldsymbol{\delta}$ ' & $250 \mathrm{~W}$ \\
\hline Delay Tolerance ' $\boldsymbol{\Delta} \boldsymbol{t}$ ' & Zero \\
\hline
\end{tabular}

\section{B. Results and Evaluation}

For results and evaluation purposes, the starting time indices ${ }^{2}$ of the detected events are considered because they initiate the events regardless whether that event is related to appliance turning ON or turning OFF. Furthermore, this paper is considering two specific appliances for evaluation purposes: $\mathrm{EV}$ and AC. These appliances are selected due to their highenergy consumption and their corresponding future impacts on the energy market. Hence, these high consumption loads can be a potential flexibility control and consumption/bills reduction lever from the grid and consumer point of view, respectively. Moreover, high consumption appliances are more viable to accurately disaggregate while acquiring the aggregated load data at low sampling rate. The EV energy consumption profile is also of interest as it has been less studied in the available literature in terms of NILM. For example, Clements-Nyns et al. [38] analyzed the impact of EV charging and found it as a significant load element specifically for smart grid system analysis.

Table III presents the results of the VSW algorithm in form of starting time indices of the detected events along with the corresponding sequence number.

TABLE III. VSW ALGORITHM RESULTS

\begin{tabular}{|c|c|c|c|}
\hline \multirow{3}{*}{$\begin{array}{l}\text { Detected } \\
\text { Events' } \\
\text { Sequence }\end{array}$} & \multicolumn{3}{|c|}{ Starting Time Indices of the Events } \\
\hline & \multirow{2}{*}{$\begin{array}{c}\text { VSW } \\
\text { Algorithm's } \\
\text { Detection }\end{array}$} & \multicolumn{2}{|c|}{ Ground Truth Data } \\
\hline & & $\mathrm{AC}$ & $\mathrm{EV}$ \\
\hline 1 & 16 & 16 & - \\
\hline 2 & 54 & 54 & - \\
\hline 3 & 96 & 95 & - \\
\hline 14 & 1101 & 1101 & - \\
\hline 15 & 1135 & 1135 & - \\
\hline 16 & 1185 & - & 1185 \\
\hline 17 & 1238 & 1238 & - \\
\hline 18 & 1272 & 1272 & - \\
\hline 19 & 1281 & - & 1281 \\
\hline 20 & 1298 & - & 1298 \\
\hline 21 & 1318 & 1317 & - \\
\hline \multicolumn{2}{|c|}{ Not Detected } & 3715 & - \\
\hline \multicolumn{2}{|c|}{ Not Detected } & 4153 & - \\
\hline 97 & 6210 & 6210 & - \\
\hline 98 & 6234 & - & 6234 \\
\hline 99 & 6247 & - & 6246 \\
\hline 100 & 6502 & - & 6502 \\
\hline 101 & 6615 & - & 6614 \\
\hline 102 & 6643 & 6643 & - \\
\hline 103 & 6686 & 6686 & - \\
\hline \multicolumn{2}{|c|}{ Not Detected } & 9780 & - \\
\hline 147 & 9837 & - & 9836 \\
\hline 148 & 9879 & 9878 & - \\
\hline 149 & 9923 & 9922 & - \\
\hline 150 & 9949 & - & 9949 \\
\hline \multicolumn{2}{|c|}{ Not Detected } & - & 10020 \\
\hline 207 & 13968 & 13968 & - \\
\hline 208 & 14030 & 14030 & - \\
\hline 209 & 14083 & 14083 & - \\
\hline 261 & 16945 & \multicolumn{2}{|c|}{ No Actual Event Occurred } \\
\hline 262 & 16970 & \multicolumn{2}{|c|}{ No Actual Event Occurred } \\
\hline 321 & 21058 & - & 21058 \\
\hline 322 & 21083 & - & 21083 \\
\hline 323 & 21103 & 21103 & - \\
\hline 324 & 21160 & - & 21159 \\
\hline
\end{tabular}

For comparison purposes, the ground-truth starting time indices of the events related to the appliances, i.e., $\mathrm{AC}$ and $\mathrm{EV}$, are also presented in Table III. It can be observed that most of the events are precisely detected by the VSW algorithm albeit with some misdetection. For example, AC and EV trigger events at time indices 3,715 and 10,020 respectively but the

\footnotetext{
${ }^{1}$ Ground-truth refers to the time indices representing when the events actually occur.

${ }^{2}$ Starting time indices of detected events presented in Tables III and IV, and that on the abscissas of Figs. 6-9 are same.
} 
VSW algorithm does not detect these events at all, leading to false negative detections. Whilst the VSW algorithm detected events at time indices 16,945 and 16,970 but no actual groundtruth events are present at these instances; this leads to false positive detections. Similarly, due to zero delay tolerance, events detected at sequence number $3,21,99 \ldots$ are not considered as true positive detections.

The results for the MAD-SW algorithm are presented in the same way. Table IV presents the starting time indices and the corresponding sequence number of the detected events by MAD-SW algorithm along with the ground-truth starting time indices of the events of the two said appliances. It is noteworthy that the ground-truth data of appliances shown in Tables III and IV are acquired for the same data identification (ID) and timeframe as presented in Table II. It can be observed from the results presented in Table IV, that most of the events are precisely detected by the MAD-SW algorithm albeit with some misdetection.

TABLE IV. MAD-SW ALGORITHM RESULTS

\begin{tabular}{|c|c|c|c|}
\hline \multirow{3}{*}{$\begin{array}{l}\text { Detected } \\
\text { Events' } \\
\text { Sequence }\end{array}$} & \multicolumn{3}{|c|}{ Starting Time Indices of the Events } \\
\hline & \multirow{2}{*}{$\begin{array}{l}\text { MAD-SW } \\
\text { Algorithm's } \\
\text { Detection }\end{array}$} & \multicolumn{2}{|c|}{ Ground Truth Data } \\
\hline & & $\mathrm{AC}$ & $\mathrm{EV}$ \\
\hline 1 & 16 & 16 & - \\
\hline 2 & 54 & 54 & - \\
\hline 3 & 95 & 95 & - \\
\hline 20 & 1298 & - & 1298 \\
\hline 21 & 1317 & 1317 & - \\
\hline 22 & 1326 & 1326 & - \\
\hline 23 & 1392 & 1392 & - \\
\hline 24 & 1432 & 1432 & - \\
\hline 76 & 4874 & - & 4874 \\
\hline 77 & 4916 & - & 4916 \\
\hline 78 & 5117 & - & 5117 \\
\hline 79 & 5218 & 5218 & - \\
\hline \multicolumn{2}{|c|}{ Not Detected } & 5361 & - \\
\hline \multicolumn{2}{|c|}{ Not Detected } & 6800 & - \\
\hline 149 & 9774 & - & 9774 \\
\hline 150 & 9780 & 9780 & - \\
\hline 151 & 9836 & - & 9836 \\
\hline 152 & 9878 & 9878 & - \\
\hline 216 & 13968 & 13968 & - \\
\hline 217 & 14011 & - & 14011 \\
\hline 218 & 14030 & 14030 & - \\
\hline 219 & 14083 & 14083 & - \\
\hline 220 & 14119 & - & 14119 \\
\hline 317 & 19960 & - & 19960 \\
\hline 318 & 19989 & - & 19989 \\
\hline 319 & 20015 & - & 20014 \\
\hline 320 & 20115 & \multicolumn{2}{|c|}{ No Actual Event Occurred } \\
\hline 321 & 20170 & 20171 & - \\
\hline 322 & 20251 & 20251 & - \\
\hline 338 & 21058 & - & 21058 \\
\hline 339 & 21083 & - & 21083 \\
\hline 340 & 21103 & 21103 & - \\
\hline 341 & 21125 & \multicolumn{2}{|c|}{ No Actual Event Occurred } \\
\hline 342 & 21159 & - & 21159 \\
\hline 343 & 21174 & \multicolumn{2}{|c|}{ No Actual Event Occurred } \\
\hline
\end{tabular}

For parameters presented in Table II, the total number of detected events by VSW and MAD-SW algorithms are 324 and 343 respectively. It is worth mentioning here that, due to space limitation Tables III and IV present a portion only of the detected events by VSW and MAD-SW respectively. Fig. 6 graphically presents a portion of the detection results by the VSW algorithm in terms of pre-processed acquired aggregated load data and the final output of the algorithm in form of starting time indices of the detected events.

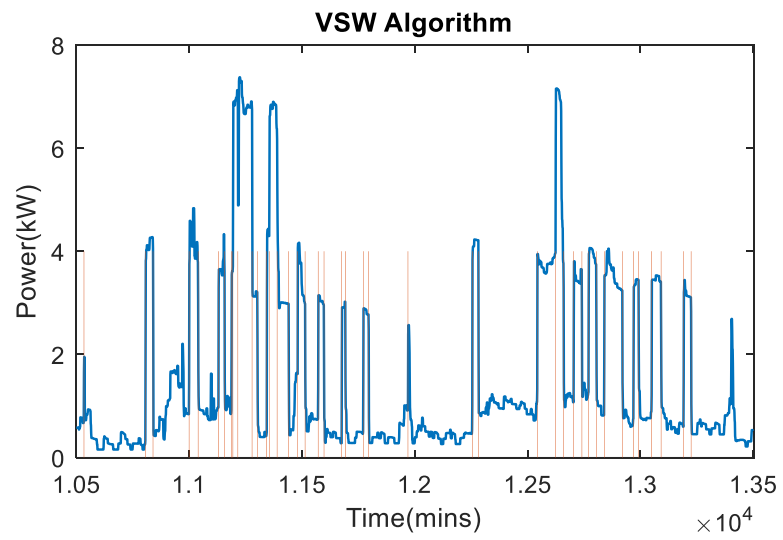

Fig. 6. Pre-processed aggregated load data (in dark cyan) and events detected by the VSW algorithm (in orange)

To further elaborate the results, Fig. 7 graphically presents the detected events and their comparison with the ground-truth signal of the appliances under consideration: AC and EV.
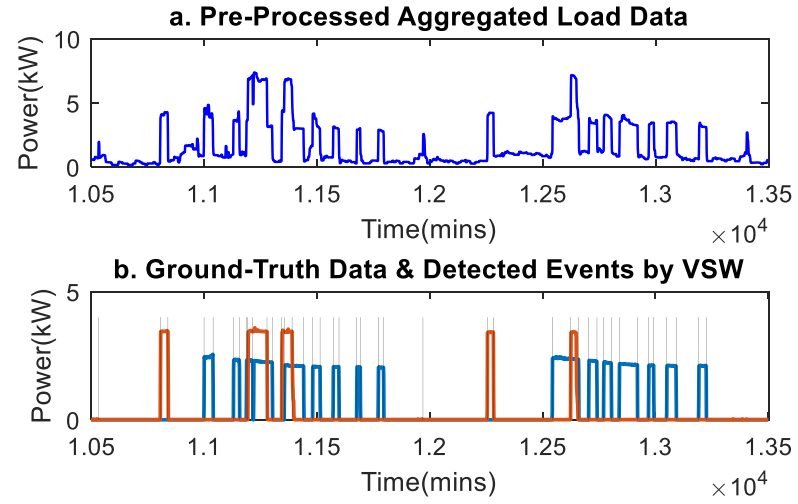

Fig. 7. (a) Pre-processed acquired aggregated load data, (b) Ground-truth signal of $\mathrm{AC}$ and $\mathrm{EV}$ appears in dark cyan and orange color respectively along with the events detected by VSW algorithm appearing in black

Likewise, Figs. 8 and 9 depict the event detection results of the MAD-SW algorithm and comparison of detected events with the ground-truth signal of the appliances respectively.

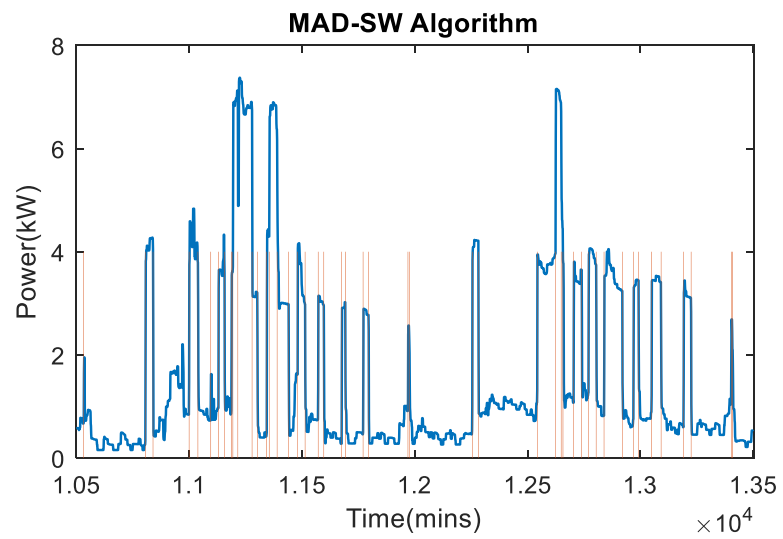

Fig. 8. Pre-processed aggregated load data (in dark cyan) and events detected by the MAD-SW algorithm (in orange) 

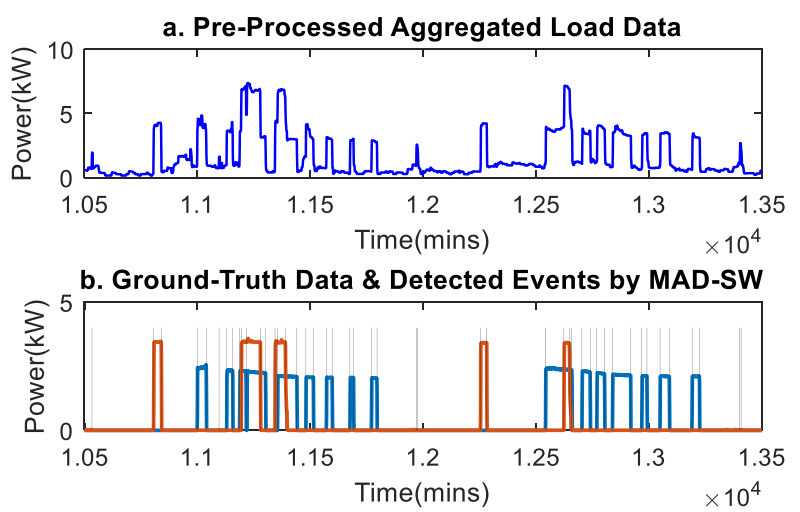

Fig. 9. (a) Pre-processed acquired aggregated data using median filtering, (b) Ground-truth signal of AC and EV appears in dark cyan and orange respectively along with the events detected by MAD-SW algorithm appearing in black color

It is evident from Figs. 6 and 8 that most of the high consumption peaks in the acquired aggregated load data are effectively detected comparatively to the lower variation in the aggregated data. This is expected and required because of the pre-defined parameters presented in Table II particularly the acquired aggregated data granularity and the threshold value [39] of $1 / 60 \mathrm{~Hz}$ and $250 \mathrm{~W}$ respectively.

For evaluating the performance of the proposed algorithms, this paper opted for widely used performance metrics, namely precision, and recall [17]. Precision is defined as the ratio between truly detected and overall detected events and is given in (5),

Precision $=\frac{\text { True Positive }}{\text { True Positive }+ \text { False Positive }}$

Recall is a measure of the detection of events occurred in reality and is given in (6),

Recall $=\frac{\text { True Positive }}{\text { True Positive }+ \text { False Negative }}$

The definitions of true positive, false positive, and false negative are given [40] in Table V.

TABLE V. TERMINOLOGIES DESCRIPTION

\begin{tabular}{|l|l|l|}
\hline $\begin{array}{l}\text { Algorithm } \\
\text { Prediction }\end{array}$ & $\begin{array}{l}\text { Actual Event } \\
\text { Occurred }\end{array}$ & $\begin{array}{l}\text { Actual Event Didn't } \\
\text { Occur }\end{array}$ \\
\hline Detected & True Positive & False Positive \\
\hline Not Detected & False Negative & True Negative \\
\hline
\end{tabular}

Based on these metrics, the performance of the proposed algorithms (as per the simulation parameters presented in Table II) are presented in Table VI.

TABLE VI. PROPOSED ALGORITHM PERFORMANCE

\begin{tabular}{|l|l|l|}
\hline $\begin{array}{l}\text { Performance } \\
\text { Metric }\end{array}$ & VSW Algorithm & $\begin{array}{l}\text { MAD-SW } \\
\text { Algorithm }\end{array}$ \\
\hline True Positive & 261 & 300 \\
\hline False Negative & 67 & 29 \\
\hline False Positive & 63 & 43 \\
\hline Precision & $80.556 \%$ & $87.464 \%$ \\
\hline Recall & $79.573 \%$ & $91.185 \%$ \\
\hline
\end{tabular}

It is evident from Table VI that due to a low number of false negative and false positive (particularly for input parameters presented in Table II), the proposed MAD-SW outperforms VSW both in terms of recall and precision.

\section{SENSITIVITY StUdies}

The proposed algorithms are comprised of different input parameters that can affect their performance, e.g., sliding window width ' $\omega$ ', delay tolerance ' $\Delta t$ ', and threshold value ' $\delta$ '. Within the scope of this paper, a sensitivity analysis has been carried out to investigate the effects of the sliding window width ' $\omega$ ' on the performance of the algorithms where the rest of the parameters are kept constant as presented in Table II.

Fig. 10 presents the corresponding results of the sensitivity analysis in terms of precision and recall performance metrics for the VSW algorithm.

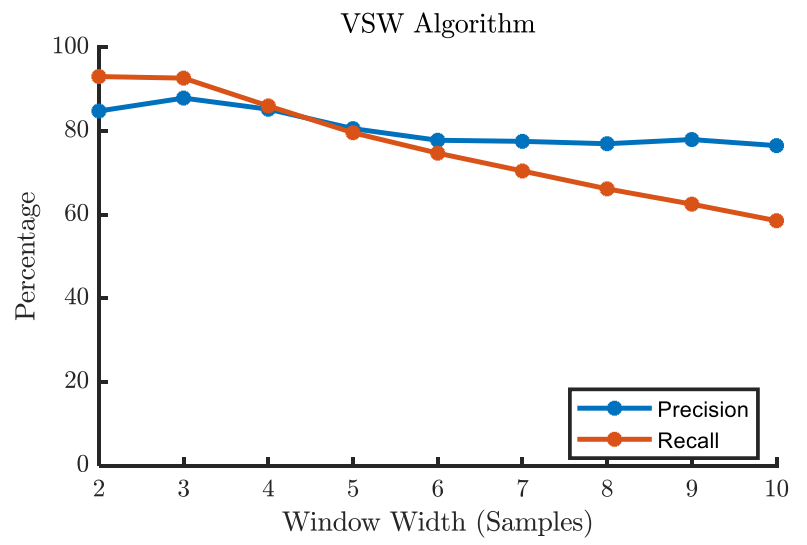

Fig. 10. Effect of ' $\omega$ ' on Performance of VSW Algorithm

The sensitivity analysis in terms of window width leads to the conclusion that overall performance (in terms of precision and recall) of the VSW algorithm is optimal at $\omega$ equals to 3 . Furthermore, the recall metric shows a continuous drop with increasing window width. This is due to the increase in the false negative detection as a function of window width.

Similarly, Fig. 11 depicts the sensitivity analysis results in terms of precision and recall for the MAD-SW algorithm.

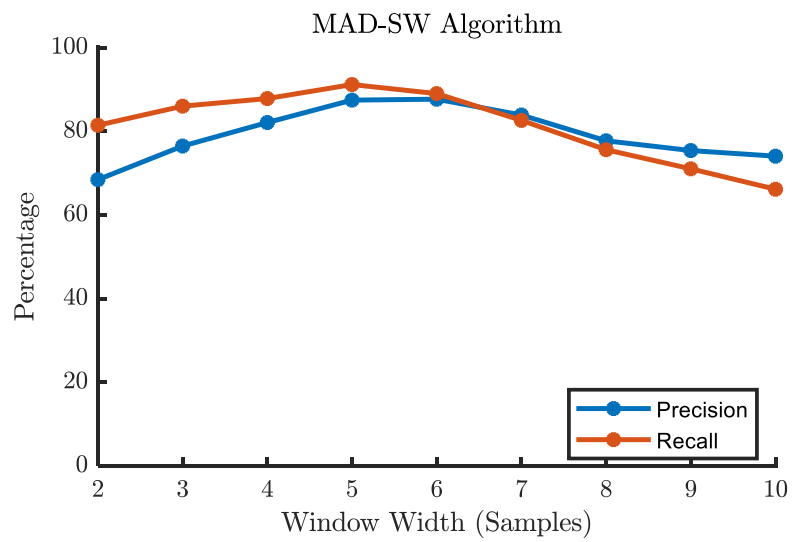

Fig. 11. Effect of ' $\omega$ ' on Performance of MAD-SW Algorithm

It is observed that the proposed MAD-SW algorithm optimally performs at a window width of 5. For $\omega>5$ the overall performance in terms of precision and recall shows a 
continuous drop. The drop is due to the rise of false positive and false negative respectively.

As a result, it is concluded that increasing the window width leads to an increase in false positive and false negative detections. Consequently, the performance of both algorithms degrades. Overall this sensitivity analysis leads to the conclusion that the optimal performances of the proposed algorithms rely on the selection of an optimal value for the input parameter $\omega$ which varies according to the algorithm.

\section{COMPARATIVE ANALYSIS}

In order to validate the proposed algorithms, a comparative analysis has been carried out where the VSW and MAD-SW algorithms are compared with the existing event detection algorithm known as High Accuracy NILM Detector (HAND) [41]. The HAND algorithm tracks the standard deviation of the aggregated load data using a moving window. The HAND algorithm has been implemented, and simulations have been carried out using the same parameters as presented in Table II. Fig. 12 graphically compares of the proposed algorithms against the HAND in terms of precision performance metrics at different window width.

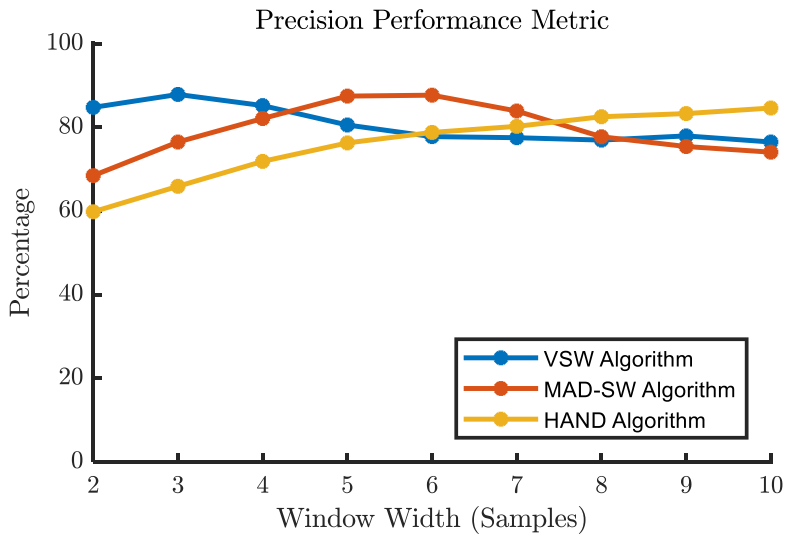

Fig. 12. Precision Performance Metric Comparison

Similarly, Fig. 13 depicts comparative results of VSW, MAD$\mathrm{SW}$, and HAND algorithms in terms of recall performance metric for different values of $\omega$.

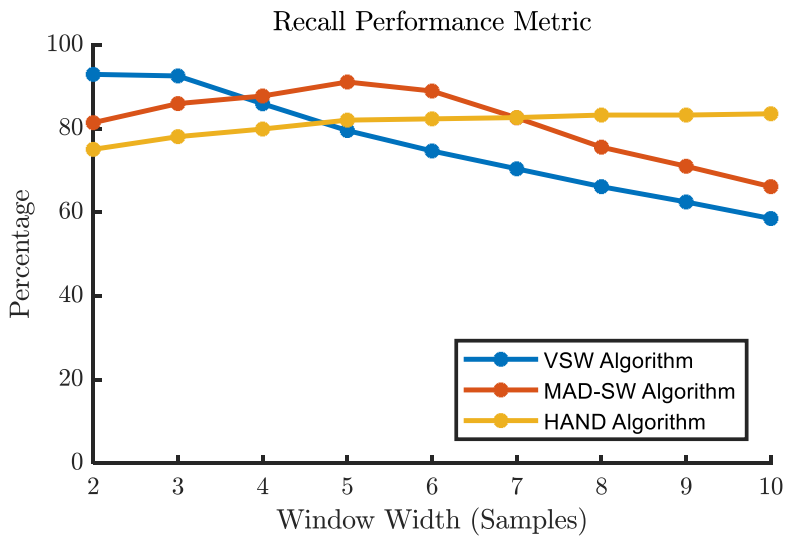

Fig. 13. Recall Performance Metric Comparison

It is evident from the comparative analysis presented in Figs. 12 and 13 that, at $\omega$ equal to 2 and 3, the VSW algorithm outperforms the other algorithms in terms of overall performance, i.e., precision and recall. For $\omega$ equals to 5 and 6 , the MAD-SW algorithm outperforms the other algorithms. The HAND algorithm has optimal results for $\omega \geq 8$ for which the proposed algorithms are not performing optimally. Furthermore, it is observed that with the increase in window width, the false positive and false negative detections by the HAND algorithm decrease thereby increasing its overall performance. It is concluded that each algorithm performs optimally at different window widths $\omega$.

\section{CONCLUSION}

This paper proposed two new event detection algorithms namely, VSW and MAD-SW algorithms, for event-based NILM systems based on statistical features and a sliding window. Beside low complexity, the proposed algorithms are computationally fast due to their iterative computational method.

Computational simulation studies were carried out on a realworld load data set and the proposed algorithms were compared against an existing event detection algorithm. It is shown that the outcome of the proposed algorithms is promising in terms of performance metrics. The window width sensitivity analysis for the proposed algorithms has shown that there is a tradeoff between the selection of an optimal window width and optimal performance. It is also observed that both proposed algorithms follow a trend of performance degradation with an increase in window width beyond the optimal window width value.

Future work will focus on the validation of the robustness of the proposed algorithms by testing them on different data sampling rate, particularly low sampling rate. To further investigate the effect of window width on the performance of the proposed algorithms, an extended sensitivity analysis will be carried out by considering different input parameters, namely, delay tolerance and threshold value. Finally, power measurement uncertainties, which could affect the event detection, would need to be investigated.

\section{ACKNOWLEDGMENT}

The authors gratefully acknowledge the research facilities and opportunities provided by Auckland University of Technology and Genesis Energy Limited. The authors also acknowledge the contribution of Callaghan Innovation for promoting the academia-industry linkages and provision of research fellowships grant.

\section{REFERENCES}

V. Amenta and G. M. Tina, "Load Demand Disaggregation Based on Simple Load Signature and User's Feedback," Energy Procedia, vol. 83, pp. 380-388, 2015.

K. Carrie Armel, A. Gupta, G. Shrimali, and A. Albert, "Is disaggregation the holy grail of energy efficiency? The case of electricity," Energy Policy, vol. 52, no. Supplement C, pp. 213-234, 2013/01/01/ 2013

A. Zoha, A. Gluhak, M. A. Imran, and S. Rajasegarar, "Nonintrusive load monitoring approaches for disaggregated energy sensing: A survey," Sensors, vol. 12, no. 12, pp. 16838-16866, 2012. Y. Wang, Q. Chen, T. Hong, and C. Kang, "Review of Smart Meter Data Analytics: Applications, Methodologies, and Challenges," arXiv preprint arXiv:1802.04117, 2018. 
[5] H.-H. Chang, L.-S. Lin, N. Chen, and W.-J. Lee, "Particle-swarmoptimization-based nonintrusive demand monitoring and load identification in smart meters," IEEE Transactions on Industry Applications, vol. 49, no. 5, pp. 2229-2236, 2013.

[6] J. Liao, G. Elafoudi, L. Stankovic, and V. Stankovic, "Power disaggregation for low-sampling rate data," in 2nd International Non-intrusive Appliance Load Monitoring Workshop, Austin, TX, 2014.

[7] Y. F. Wong, Y. A. Şekercioğlu, T. Drummond, and V. S. Wong, "Recent approaches to non-intrusive load monitoring techniques in residential settings," in Computational Intelligence Applications In Smart Grid (CIASG), 2013 IEEE Symposium on, 2013, pp. 73-79: IEEE.

[8] J. Liang, S. K. Ng, G. Kendall, and J. W. Cheng, "Load signature study_Part I: Basic concept, structure, and methodology," IEEE transactions on power Delivery, vol. 25, no. 2, pp. 551-560, 2010.

[9] S. R. Shaw, S. B. Leeb, L. K. Norford, and R. W. Cox, "Nonintrusive load monitoring and diagnostics in power systems," IEEE Transactions on Instrumentation and Measurement, vol. 57, no. 7, pp. 1445-1454, 2008.

[10] D. Egarter and W. Elmenreich, "Load disaggregation with metaheuristic optimization," Energieinformatik, Karlsruhe, Germany, 2015.

[11] Y.-H. Lin and M.-S. Tsai, "An advanced home energy management system facilitated by nonintrusive load monitoring with automated multiobjective power scheduling," IEEE Transactions on Smart Grid, vol. 6, no. 4, pp. 1839-1851, 2015.

[12] G. W. Hart, "Nonintrusive appliance load monitoring," Proceedings of the IEEE, vol. 80, no. 12, pp. 1870-1891, 1992.

[13] H. Altrabalsi, V. Stankovic, J. Liao, and L. Stankovic, "Lowcomplexity energy disaggregation using appliance load modelling," AIMS Energy, vol. 4, no. 1, pp. 884-905, 2016.

[14] H. Altrabalsi, J. Liao, L. Stankovic, and V. Stankovic, "A lowcomplexity energy disaggregation method: Performance and robustness," in Computational Intelligence Applications in Smart Grid (CIASG), 2014 IEEE Symposium on, 2014, pp. 1-8: IEEE.

[15] G. W. Hart, Nonintrusive Appliance Load Data Acquisition Method: Progress Report. MIT Energy Laboratory, 1984.

[16] M. Zeifman and K. Roth, "Nonintrusive appliance load monitoring: Review and outlook," IEEE transactions on Consumer Electronics, vol. 57, no. 1, 2011.

[17] A. Faustine, N. H. Mvungi, S. Kaijage, and K. Michael, "A Survey on Non-Intrusive Load Monitoring Methodies and Techniques for Energy Disaggregation Problem," arXiv preprint arXiv:1703.00785, 2017.

[18] C. Klemenjak and P. Goldsborough, "Non-Intrusive Load Monitoring: A Review and Outlook," arXiv preprint arXiv:1610.01191, 2016.

[19] "Low Cost NIALMS Technology," Electric Power Research Institute, Technical Report TR-198918-V1, Sept. 1997, vol. 1.

[20] L. Farinaccio and R. Zmeureanu, "Using a pattern recognition approach to disaggregate the total electricity consumption in a house into the major end-uses," Energy and Buildings, vol. 30, no. 3, pp. 245-259, 1999

[21] M. L. Marceau and R. Zmeureanu, "Nonintrusive load disaggregation computer program to estimate the energy consumption of major end uses in residential buildings," Energy conversion and management, vol. 41, no. 13, pp. 1389-1403, 2000.

[22] A. Rahimpour, H. Qi, D. Fugate, and T. Kuruganti, "Non-intrusive energy disaggregation using non-negative matrix factorization with sum-to-k constraint," IEEE Transactions on Power Systems, vol. 32, no. 6, pp. 4430-4441, 2017.

[23] T. Bernard and M. Marx, "Unsupervised learning algorithm using multiple electrical low and high frequency features for the task of load disaggregation," in Proceedings of the 3rd International Workshop on NILM, Vancouver, BC, Canada, 2016, pp. 14-15.

[24] K. D. Anderson, M. E. Bergés, A. Ocneanu, D. Benitez, and J. M. Moura, "Event detection for non intrusive load monitoring," in IECON 2012-38th Annual Conference on IEEE Industrial Electronics Society, 2012, pp. 3312-3317: IEEE.

[25] M. B. Figueiredo, A. De Almeida, and B. Ribeiro, "An experimental study on electrical signature identification of non-intrusive load monitoring (nilm) systems," in International Conference on Adaptive and Natural Computing Algorithms, 2011, pp. 31-40: Springer.
[26] J. Z. Kolter and T. Jaakkola, "Approximate inference in additive factorial hmms with application to energy disaggregation," in Artificial Intelligence and Statistics, 2012, pp. 1472-1482.

[27] H. Kim, M. Marwah, M. Arlitt, G. Lyon, and J. Han, "Unsupervised disaggregation of low frequency power measurements," in Proceedings of the 2011 SIAM International Conference on Data Mining, 2011, pp. 747-758: SIAM.

[28] D. Egarter, V. P. Bhuvana, and W. Elmenreich, "PALDi: Online load disaggregation via particle filtering," IEEE Transactions on Instrumentation and Measurement, vol. 64, no. 2, pp. 467-477, 2015.

[29] L. De Baets, J. Ruyssinck, D. Deschrijver, and T. Dhaene, "Event detection in NILM using cepstrum smoothing," in 3rd International Workshop on Non-Intrusive Load Monitoring, 2016, pp. 1-4.

[30] J. Alcalá, J. Ureña, Á. Hernández, and D. Gualda, "Event-based energy disaggregation algorithm for activity monitoring from a single-point sensor," IEEE Transactions on Instrumentation and Measurement, vol. 66, no. 10, pp. 2615-2626, 2017.

[31] M. Azaza and F. Wallin, "Evaluation of classification methodologies and Features selection from smart meter data," Energy Procedia, vol. 142, pp. 2250-2256, 2017/12/01/ 2017.

[32] B. Wild, K. S. Barsim, and B. Yang, "A new unsupervised event detector for non-intrusive load monitoring," in Signal and Information Processing (GlobalSIP), 2015 IEEE Global Conference on, 2015, pp. 73-77: IEEE.

[33] C. Laughman et al., "Power signature analysis," IEEE power and energy magazine, vol. 99, no. 2, pp. 56-63, 2003.

[34] A. A. Girmay and C. Camarda, "Simple event detection and disaggregation approach for residential energy estimation," in Workshop on Non-Intrusive Load Monitoring (NILM), 2016 Proceedings of the 3rd International, 2016.

[35] "Pecan Street Inc. Dataport 2018,".

[36] O. Parson et al., "Dataport and NILMTK: A building data set designed for non-intrusive load monitoring," in Signal and Information Processing (GlobalSIP), 2015 IEEE Global Conference on, 2015, pp. 210-214: IEEE.

[37] K. Basu, V. Debusschere, S. Bacha, A. Hably, D. Van Delft, and G. J. Dirven, "A generic data driven approach for low sampling load disaggregation," Sustainable Energy, Grids and Networks, vol. 9, pp. 118-127, 2017.

[38] K. Clement-Nyns, E. Haesen, and J. Driesen, "The impact of charging plug-in hybrid electric vehicles on a residential distribution grid," IEEE Transactions on power systems, vol. 25, no. 1, pp. 371380,2010

[39] G. Elafoudi, L. Stankovic, and V. Stankovic, "Power disaggregation of domestic smart meter readings using dynamic time warping," in Communications, Control and Signal Processing (ISCCSP), 2014 6th International Symposium on, 2014, pp. 36-39: IEEE.

[40] M. Aiad and P. H. Lee, "Unsupervised approach for load disaggregation with devices interactions," Energy and Buildings, vol. 116, pp. 96-103, 2016.

[41] M. N. Meziane, P. Ravier, G. Lamarque, J.-C. Le Bunetel, and Y Raingeaud, "High accuracy event detection for Non-Intrusive Load Monitoring," in Acoustics, Speech and Signal Processing (ICASSP), 2017 IEEE International Conference on, 2017, pp. 2452-2456: IEEE.

\section{BIOGRAPHIES}

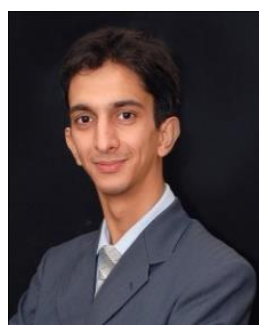

Attique Ur Rehman received the B.E. degree in electrical engineering from Air University, Islamabad, Pakistan in 2009, and the M.Sc. degree in electrical engineering, information technology and computer engineering from RWTH Aachen University, Germany in 2013. Currently, he is pursuing his Ph.D. degree from Auckland University of Technology, New Zealand.

His employment experience included both teaching and field work as Research Associate at Faculty of Electrical Engineering, GIK Institute of Engineering Sciences and Technology, Pakistan, and Engineer Switching at Pakistan Telecommunication Company Limited respectively. His research interests lie around Integration of ICT and Energy Systems, and Energy Efficiency and Conservation.

Mr. Rehman is a recipient of Callaghan Innovation R\&D StudentFellowship Grant, and AUT Doctoral Fee Scholarship. 


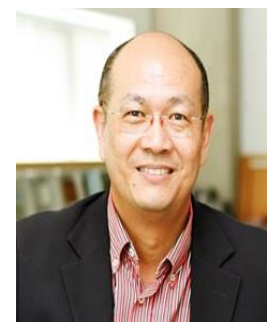

Tek Tjing Lie received his Master of Science and $\mathrm{Ph} . \mathrm{D}$. degrees in electrical engineering from Michigan State University, USA in 1988 and 1992 respectively. He is senior member of IEEE.

He had worked in Nanyang Technological University, Singapore. Currently he is working as a professor and a Deputy Head of School of Engineering, Computer and Mathematical Sciences. Auckland University of Technology, New Zealand. $\mathrm{He}$ is also a Director of Center for Energy \& Power Engineering. His research interests are in the fields of Power System Planning, Operation and Control, Deregulated Electric Power Markets, Energy Management, Renewable Energy and Smart Grids.

Prof. Lie has regularly attracted external funding for projects with industry and supervises several postgraduate students on a wide range of research topics.

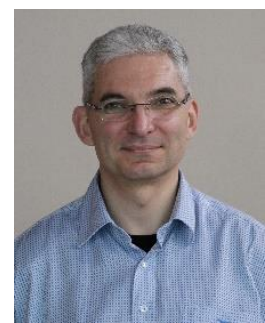

Brice Vallès received his Ph.D. degree in fluid mechanics from Norwegian University of Science and Technology, Norway in 2001.

He has more than 15 years of experience in data science and energy sector that includes Schlumberger and IRIS in Norway. Since 2010 within New Zealand, he worked in different roles at UniServices, Inland Revenue and Genesis. Currently, he is working as a Wholesale Analytics Manager at Genesis Energy Ltd. New Zealand. His keen interest is in sequential data assimilation to update simulation models in real time.

Dr. Vallès is also actively involved in business development, strategy, planning, and marketing.

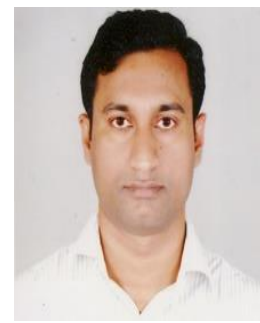

Dr. Shafiqur Rahman Tito obtained his Ph.D. degree in electrical engineering from Auckland University of Technology in 2016.

His employment experience included 12 years of teaching at different national and international level. $\mathrm{He}$ also worked as a Program Leader of Electrical Engineering at International College of Auckland, New Zealand. Currently, he is working as a Lecturer at Manukau Institute of Technology, New Zealand. His fields of research included Energy Optimization and Hybrid Renewable Energy Systems.

Dr. Rahman was a recipient of AUT Vice Chancellor Doctoral Scholarship. 